\title{
Physical and dynamical characterization of (5201) Ferraz-Mello, a possible extinct Jupiter family comet ${ }^{\star}$
}

\author{
J. M. Carvano ${ }^{1}$, S. Ferraz-Mello ${ }^{2}$, and D. Lazzaro ${ }^{1}$ \\ 1 Observatório Nacional (COAA), rua Gal. José Cristino 77, São Cristóvão, CEP20921-400 Rio de Janeiro RJ, Brazil \\ e-mail: carvano@on.br \\ 2 IAG, Universidade de São Paulo, rua do Matão 1226 CEP 05508-900 São Paulo SP, Brazil
}

Received 20 March 2008 / Accepted 13 June 2008

\begin{abstract}
Context. The subject of asteroids in cometary orbits (ACOs) has been of growing interest lately. These objects have the orbital characteristics typical of comets, but are asteroidal in appearance, i.e., show no signs of a coma at any part of their orbits. At least a fraction of these objects are thought to be comets that have either exhausted all their volatile content or developed a refractory crust that prevents sublimation. In particular, the asteroid (5201) Ferraz-Mello has, since its discovery, been suspected to be an extinct Jupiter family comet due to the peculiar nature of its orbit.

Aims. The aim of this work is to put constraints on the possible origin of (5201) Ferraz-Mello by means of spectroscopic characterization and a study of the dynamics of this asteroid

Methods. We used the SOAR Optical Imager (SOI) to obtain observations of (5201) Ferraz-Mello using four SDSS filters. These observations were compared to asteroids listed in the Sloan Moving objects catalog and also to photometry of cometary nuclei, Centaurs, and TNOs. The orbital evolution of (5201) Ferraz-Mello and of a sample of asteroids and comets that are close to that object in the $a-e$ plane were simulated using a pure $N$-body code for 4000 years forward and 4000 years backward in time.

Results. The reflectance spectrum obtained from its colors in the SDSS system is unusual, with a steep spectral gradient that is comparable to TNOs and Centaurs, but with an increase in the reflectance in the $g$ band that is not common in those populations. A similar behavior is seen in cometary nuclei that were observed in the presence of a faint dust coma. The dynamical results confirm the very chaotic evolution found previously and its dynamical similarity to the chaotic evolution of some comets. The asteroid is situated in the very stochastic layer at the border of the 2/1 resonance, and it has a very short Lyapunov time (30-40) years. Together, the spectral characteristes and the dynamical evolution suggest that (5201) Ferraz-Mello is a dormant or extinct comet.
\end{abstract}

Key words. minor planets, asteroids - comets: general

\section{Introduction}

Asteroids and comets are traditionally considered to be members of two distinct populations, both remnants of the protosolar disk that differ mainly by the region of the disk where they were formed. Asteroids were formed in the inner, hotter part of the disk and are therefore volatile-poor; comets, on the other hand, were formed in their outer, icy part of the solar nebula and thus became rich in volatiles. From an observational point of view, a comet is defined as a body that, when coming close enough to the Sun, develops a coma produced by the sublimation and outgassing of volatile material. Indeed, $\mathrm{H}_{2} \mathrm{O}$ ice, which is the most common volatile in comets, will start sublimating at distances $<4 \mathrm{UA}$, which correspond to the outer region of the main asteroid belt. Also, the two populations are much different from a dynamical point of view. The most remarkable distinction is the value of the Tisserand invariant (Kresak 1979). This parameter, an integral of motion of the restricted problem Sun-Jupiter-object, is defined by the relation, $T=\left(a_{J} / a\right)+2 \cos I \sqrt{\left(a / a_{\mathrm{J}}\right)\left(1-e^{2}\right)}$, where $a$ and $a_{\mathrm{J}}$ are the

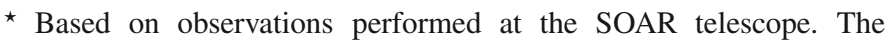
SOAR Telescope is a joint project of: Conselho Nacional de Pesquisas Científicas e Tecnológicas CNPq-Brazil, The University of North Carolina at Chapel Hill, Michigan State University, and the National Optical Astronomy Observatory. semi-major axes of the orbits of the object and Jupiter, respectively, $e$ and $I$ are the eccentricity and the inclination of the object's orbit (relative to the orbital plane of Jupiter), respectively. Kresak (1979) noted that most comets have $T<3$, while most asteroids have $T>3$.

Nowadays, several lines of evidence suggest the possibility of a genetic link between comets and asteroids: (a) the existence of icy objects, such as the trans-Neptunians and Centaurs, that are unlikely to develop a coma, since they never come close enough to the Sun; (b) the discovery that sublimation can stop on comets, either by the depletion of the volatile material or by the growth of a surface crust of refractory material (Rickman et al. 1990); (c) the observation of objects in the main belt of asteroids that present temporal burst of activity, such as (7968) ElstPizarro, P/2005 U1 Read and (118401) 1999 RE 70 (Elst et al. 1996; Hsieh et al. 2004; Hsieh \& Jewitt 2006); (d) the association of meteor showers to asteroids, such as (3200) Phaeton and others (Whipple 1983; Jenniskens 2004; Williams et al. 2004; Babadzhanov \& Kokhirova 2007); (e) the presence of asteroids with no visible coma in orbits with Tisserand parameter smaller than 3 (Kresak 1979; Hahn \& Rickman 1985; Binzel et al. 2001; Jewitt 2002; Fernández et al. 2005; DeMeo \& Binzel 2006; Alvarez-Candal \& Licandro 2006; Licandro et al. 2008). These objects are usually referred to as ACOs (asteroids in cometary orbits). 
Table 1. Geometrical configurations of the observations.

\begin{tabular}{cccc}
\hline \hline Night & $R(\mathrm{AU})$ & $\Delta(\mathrm{AU})$ & Phase angle \\
\hline May 24 2007 & 3.10 & 2.10 & $2.78^{\circ}$ \\
Jun. 26 2007 & 3.27 & 2.36 & $9.19^{\circ}$ \\
\hline
\end{tabular}

In the present study we focus on one special object, (5201) Ferraz-Mello. Since its discovery, the asteroid (5201) Ferraz-Mello (1983 XF) has puzzled astronomers by its orbital characteristics that are extremely unusual for an asteroid (Bowell et al. 1984; Benest et al. 1985; Hahn \& Rickman 1985). The suspicions that it should be a comet were considerable. A long exposure was taken on January 2nd 1984 by Everhart using a $0.4 \mathrm{~m}$ telescope at the Chamberlin Observatory's Field Station, but it failed to show any trace of coma or tail (Bowell et al. 1984).

Before the last opposition, the need to have physical observations of this asteroid was emphasized by Weissman et al. (1989), warning that it would reach its maximum brightness in 1994 at $V=16.0$ mag. However, no physical observations were reported at that very favorable opposition, nor at the subsequent one in 2001. Weissman et al. (2002) emphasized again that because of its dynamical regime this asteroid could be a possible extinct Jupiter family comet but that nothing could be said without physical studies. In 2003, we studied the orbital evolution of the asteroid using the elements available after the 2001 opposition and posted a web page (http: //www . astro.iag.usp. $\mathrm{br} /$ sylvio/a5201/Dec2003.html) averting the next two oppositions around October 2005 and June 2007 (not as favorable as in 1994, since the maximum brightness predicted was in the range $V=18.0-19.0 \mathrm{mag}$ ). No physical observations were recorded in 2005 .

The opposition of (5201) Ferraz-Mello in 2007 allowed us to make the first physical observations of the object, and this encouraged us to perform a deeper dynamical study involving not only this asteroid but also a sample of objects in the same region of the Solar System. The aim of the present work is, therefore, to see, if from an observational and dynamical point of view, (5201) Ferraz-Mello could be an extinct Jupiter family comet. In the next section, we present the description of the observations and reduction, while the taxonomic interpretation of the data is given in Sect. 3. In Sects. 4 and 5 are given the dynamical study aconcerning (5201) Ferraz-Mello and asteroids and comets in similar orbits, respectively. The main results are discussed in the last section.

\section{Observations and data reduction}

Photometric observation of the asteroid (5201) Ferraz-Mello with the $g, r, i$, and $z$ filters of the SDSS system was acquired with the SOAR Optical Imager (SOI), mounted on the $4 \mathrm{~m}$ SOAR telescope on Cerro Pachon in Chile, during service-mode observations. The asteroid was first observed during engineering time on May 24, 2007 and again during science time on June 26,2007 . Both nights were photometric, but problems with the tracking led to the loss of the z-filter data during the engineering run. Details on the geometry of the observations on both nights are given in Table 1. On both nights the photometric standard stars SA109 71 and SA109 381 were observed on at least three different air masses on all filters to allow the correction of the atmospheric extinction. No dithering was used during the observations. The exposition times for the $g, r, i$, and $z$ filters for (5201) Ferraz-Mello were, respectively, 240, 300, 360, and $1920 \mathrm{~s}$.
Table 2. Calibrated SDSS magnitudes of (5201) Ferraz-Mello.

\begin{tabular}{cccc}
\hline \hline UT (start) & Filter & Mag. & $\Delta t$ (h.) \\
\hline 2007-05-24T04:40:49.1 & $\mathrm{g}$ & $19.69 \pm 0.04$ & 0.00 \\
2007-05-24T04:50:38.6 & $\mathrm{r}$ & $19.04 \pm 0.03$ & 0.07 \\
2007-05-24T04:45:17.4 & $\mathrm{i}$ & $18.65 \pm 0.04$ & 0.16 \\
2007-06-26T01:37:31.8 & $\mathrm{z}$ & $19.22 \pm 0.02$ & 0.00 \\
2007-06-26T02:35:55.8 & $\mathrm{g}$ & $20.39 \pm 0.09$ & 0.97 \\
2007-06-26T02:46:15.7 & $\mathrm{i}$ & $19.50 \pm 0.09$ & 1.15 \\
2007-06-26T02:55:28.4 & $\mathrm{r}$ & $19.82 \pm 0.05$ & 1.30 \\
\hline
\end{tabular}

Table 3. SDSS colors of (5201) Ferraz-Mello.

\begin{tabular}{cccc}
\hline \hline Color index & Night 1 & Night 2 & Mean \\
\hline$g-r$ & $0.66 \pm 0.05$ & $0.57 \pm 0.10$ & $0.62 \pm 0.06$ \\
$r-i$ & $0.39 \pm 0.05$ & $0.32 \pm 0.10$ & $0.36 \pm 0.06$ \\
$r-z$ & - & $0.60 \pm 0.05$ & - \\
\hline
\end{tabular}

The data reduction consisted of overscan, bias and flat field corrections for all filters plus fringing correction for the $i$ and $z$ filters. After reduction the profile of (5201) in the images were indistiguishable from the profile of the stars in the observing run, with no visible coma. The instrumental magnitudes for the asteroids and standard stars were determined through aperture photometry, using photometric growth curves (Howell 1989) to determine the optimum aperture. The observations of the standard stars were then used to calculate the zero point, extinction coefficient, and color term of each night, with the respective uncertainties. The calibrated magnitudes of (5201) Ferraz-Mello are shown on Table 2. This table also shows the time intervals between the start of the observations at each filter. Even if the rotation period of (5201) Ferraz-Mello is not known, the relatively short intervals between the filter acquisitions suggest that the rotational variation must not bear an effect too strong on the calculated color. The SDSS colors for each night and their mean values are given in Table 3.

\section{Spectral reflectance and classification}

To obtain a taxonomic classification of (5201) Ferraz-Mello, the SDSS colors $(g-r),(r-i)$, and $(r-z)$ were converted to flux by removing the solar component using the solar colors from Ivezić et al. (2001), and then applying the usual flux-magnitude transformation (Roig \& Gil-Hutton 2006). The resulting reflectance is normalized on the $r$ band and are shown in Fig. 1. The data of the two nights show good consistency, falling within each other's error bars. Both datasets reveal a featureless spectra (i.e., no evidence of a fall in reflectivity due to the first pyroxene band at $0.9 \mu \mathrm{m})$, with a steep spectral slope.

This kind of spectra is observed on main belt D-type asteroids, Trojans, Centaurs, TNOs and cometary nuclei. Each of these types of objects present typical (although overlapping) ranges of spectral inclination, which can be characterized by the normalized reflectivity gradient (Jewitt 2002):

$S^{\prime}=(\mathrm{d} S / \mathrm{d} \lambda) / \bar{S}$

where $S$ the reflectivity and $\bar{S}$ the average reflectivity in the wavelength range used to calculate the gradient. In Table 4 we compare the value of $S$ calculated for (5201) Ferraz-Mello with the values compiled by Jewitt (2002) for each of the aforementioned group plus a sample of near-Earth ACOs, which are considered by the author as possible dead comets. The uncertainties quoted in the table for each population is three times the 


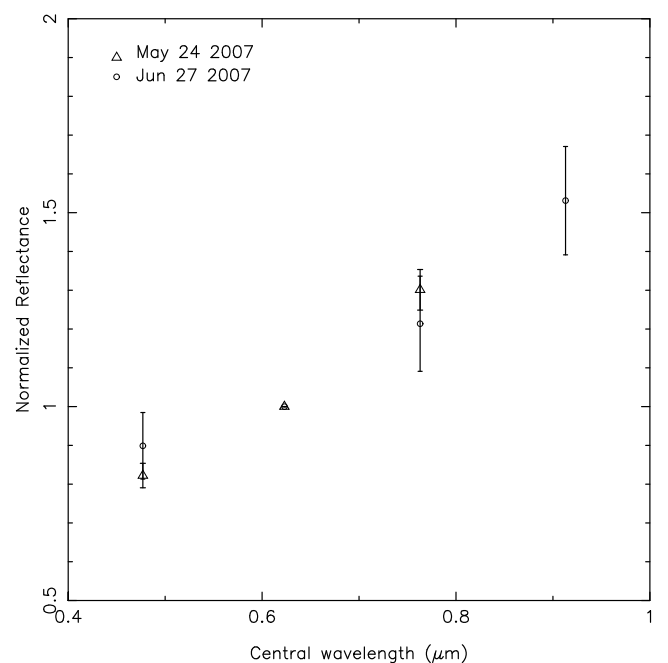

Fig. 1. Normalized reflectance spectrum for (5201) Ferraz-Mello.

Table 4. Normalized reflectivity gradients $\left[\%(1000 \AA)^{-1}\right]$.

\begin{tabular}{cc}
\hline \hline Object or Group & $S^{\prime}$ \\
\hline Dead Comets & $7.2 \pm 6$ \\
Nuclei & $8.3 \pm 8.4$ \\
D-types & $8.8 \pm 1.5$ \\
Trojans & $9.6 \pm 2.7$ \\
Centaurs & $17 \pm 15$ \\
TNOs & $22.9 \pm 5.4$ \\
(5201) Ferraz-Mello & $12.6 \pm 3$ \\
\hline
\end{tabular}

variance observed among the members of the population. For (5201) Ferraz-Mello, we computed the gradient in reflectivity as the angular coefficient of a linear fit. This comparison suggests that the spectral inclination of (5201) Ferraz-Mello is compatible with what is observed among Centaurs, dead comets, and cometary nuclei, but higher than most of the main belt D-types and trojans, and lower than the majority of the TNOs.

To obtain a more comprehensive picture of how typical the spectrum of (5201) Ferraz-Mello is, we compared it to a subsample of the solar system objects observed by the Sloan Digital Sky Survey (Gunn 1999). To do so, we calculate the principal components of a sample of 24643 spectra of objects listed in the SDSS Moving Object Catalog (Juric et al. 2007) in the filters $g$, $r, i$, and $z$, which have uncertainties comparable with or smaller than our observations, plus a sample of 125 Centaurs and TNOs and 13 cometary nuclei. The spectra of the Centaurs, TNOs, and Comets were obtained by first converting the BVRI colors to $g$, $r, i, z$ colors, using the transformation given in Jordi et al. (2006) and then converting it to reflectivity. The colors for Centaurs and TNOS were obtained from the compilation available in the PDS repository (Neese 2006b), and whenever multiple observations of the same object were available we used the average colors. For the cometary nuclei, we used the BVRI colors from Jewitt (2002) and Snodgrass et al. (2007).

The resulting distributions in the two first principal components are shown in Fig. 2. The asteroids fall on a V-shaped cluster around the origin of the plot. The position of (5201) Ferraz-Mello is marked by a black dot enclosed by a box representing the uncertainties of the observations propagated into principal component space. For reference Fig. 2a shows the position of the asteroids with unambiguous taxonomic classification in either Tholen (Tholen \& Barucci 1989) or Bus

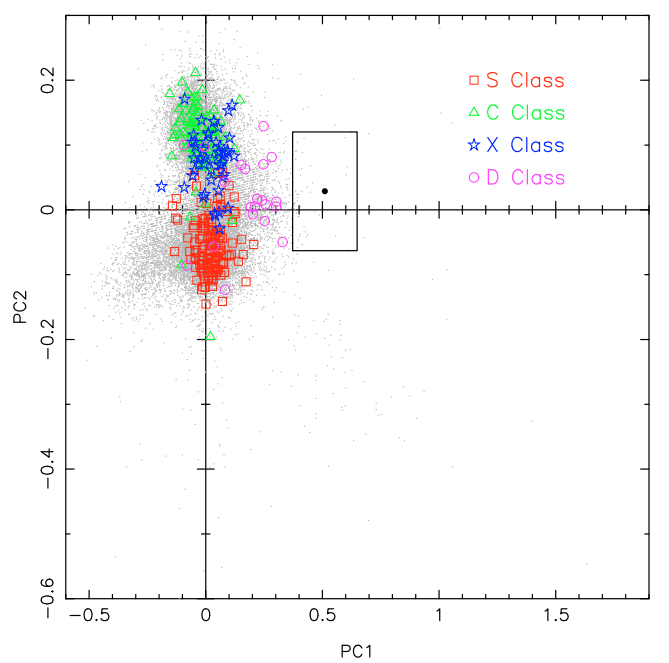

(a)

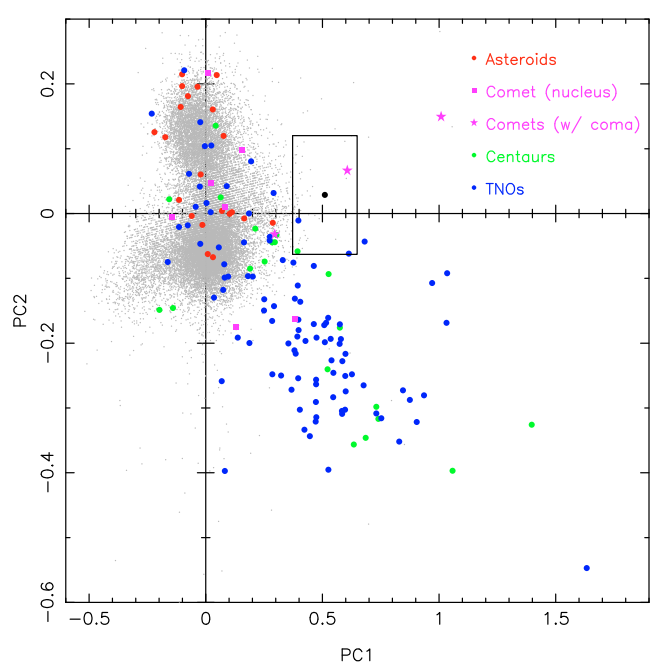

(b)

Fig. 2. Principal components plot. (5201) Ferraz-Mello is represented by a black dot, and the propagated uncertainty of its spectra is delimited by a box. a) Position of the asteroids belonging to the C, D, X, and $\mathrm{S}$ classes. b) Position of TNOs, Centaurs, Jupiter family nuclei, and asteroids in the same dynamical region as (5201) Ferraz-Mello (see Sect. 4).

(Bus \& Binzel 2002) taxonomies, compiled by Neese (2006a), that were also observed by the SDSS. We plot only the classes C, X, S, and D (Bus' subclasses are considered within their complexes.) The featureless $\mathrm{C}, \mathrm{X}$, and D classes stretch from the upper left boundary of the main cluster (C-types, with spectral gradient from slightly negative to neutral) to its lower right boundary (D-types, relatively high positive spectral gradients). Figure $2 b$ shows the same plot, but highlighting objects of four dynamical groups: TNOs, Centaurs, Jupiter family cometary nuclei, and asteroids in the same dynamical region as (5201) Ferraz-Mello. The last follow the definition given in Sect. 4, but allowing any Tisserand parameter. From their position on the principal component space, the asteroids in this region are mainly a mixture of C-, X-, and D-types, i.e., featureless with spectral gradients ranging from slightly negative to relatively high positive values. The Centaurs and TNOs, on the other hand, stretch from the loci of the C-type asteroids to beyond the D-type loci, reflecting the higher values of spectral gradients that are 
seen in these populations. From these figures it is clear that the spectra of (5201) Ferraz-Mello is unusual for a main belt object, since it plots well outside the cluster formed by the SDSS asteroids, and it also falls outside the main trend for TNOs, Centaurs and Jupiter Family nuclei.

To conclude this analysis, we also searched for all ACOs in the neighborhood of (5201) Ferraz-Mello that have a taxonomic classification and a low-resolution spectra. Among these there are four C-type, three P-type, four D-type and one T-type. The nearest to (5201) Ferraz-Mello are (3688) Navajo (P-type) and $1999 \mathrm{SE}_{10}$ (D-type) (Licandro et al. 2008; Demeo \& Binzel 2008). On the other hand, there are just three comets in the region that do have a published spectra: 9P/Tempel 1, 10P/Tempel, 46P/Wirtanen, and 107P/Wilson-Harrington. All of these have a spectra similar to (5201) Ferraz-Mello, even if not as red. This confirms the above finding that this asteroid has a redder spectra than the mean D-type asteroids and comets.

Although unusual, the spectrum of Ferraz-Mello plots close to a number of objects in the principal component space. If we consider only objects that lie within the area delimited by the propagated uncertainties of our observations, we have a total of 46 main belt objects, two TNOs, one Centaurs, and one comet nucleus with spectra that resemble Ferraz-Mellos's within error bars. Figure 3 a compares the spectra of (5201) Ferraz-Mello with the best matching TNOs (2001 KB77 and 1998 WW24), Centaur (2001 BL41) and comet nucleus (40P/Väisälä 1) and the best matching asteroids (2000 EO20). For comparison this figure also shows the the spectra of a typical D-type asteroid and the one of a "typical" TNO, taken here as a TNO with similar spectral gradient as (5201) Ferraz-Mello but which lies within the main trend for TNOs in principal component space.

The closest spectral match with (5201) Ferraz-Mello is with the two asteroids. However the spectral gradient of all three asteroids is steeper than a typical main belt D-type asteroid, but fall comfortably in the range observed for Centaurs and TNOs. On the other hand, (5201) Ferraz-Mello differs from both typical D-type asteroids and "typical" Centaurs and TNOs by the behavior of the flux at the $g$ filter, which for Ferraz-Mello lies above the linear trend extrapolated from the flux at the filters $r$ and $i$, while typical D-types and TNOs present a flux at the $g$ band below or at that trend. Indeed, an insight on the meaning of PC1 and PC2 for this type of objectcan be obtained from the comparison of the reflectance and principal components of the TNO 1998 WW24 with comet 40P/Väisälä 1. Both objects have essentially the same reflectance on the filters $i$, and $z$, but present very different reflectances at the $g$ filter. Likewise, both present virtually the same value of $\mathrm{PC} 1$ but very different values of PC2. Therefore (5201) Ferraz-Mello and the objects with similar spectra are unusual when compared with Centaurs and TNOs due to a relative enhancement of their reflectance in the $g$ band.

One way to explain such peculiarity is to assume an unusual composition for these objects. Another possible explanation is suggested because the best matching comet nuclei, 40P/Väisälä 1, presented a faint dust coma at the instant of the observation (Snodgrass et al. 2007). Indeed, Fig. 3b show the derived reflectance spectra of the comet nuclei that were observed with a dust coma. All present the same characteristic relative enhancement of the reflectance at the $g$ filter. If that is the case, (5201) Ferraz-Mello should have a faint dust coma, even if it could not be directly detected on our observations. This could also be true for the other objects with similar spectra. These objects all have $T_{j}>3$ and are scattered throughout the main belt, from its inner portion to the Trojan region, but they seem to

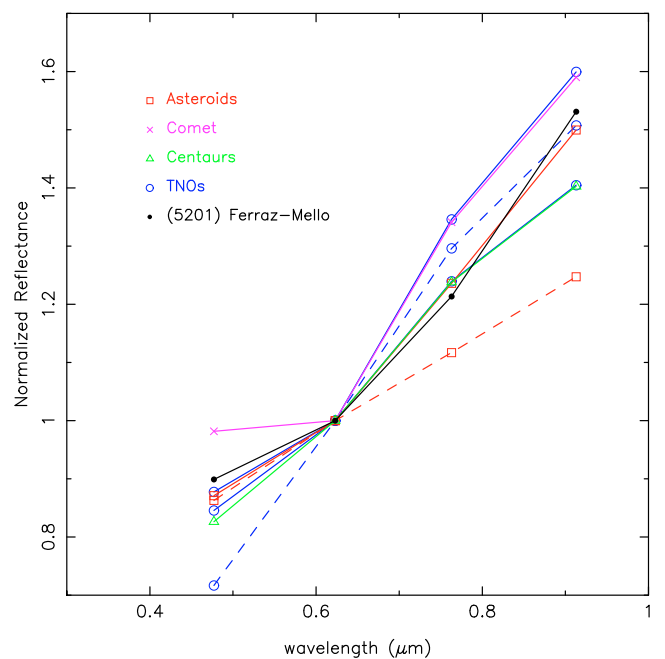

(a)

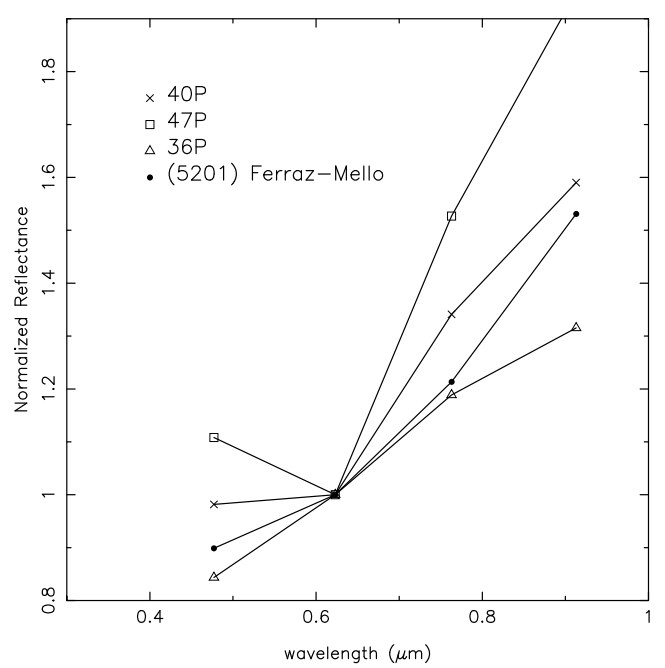

(b)

Fig. 3. Comparison of the reflectance spectra of (5201) Ferraz-Mello with a) the best matching TNOs, Centaurs, cometary nuclei and asteroid (solid lines), plus a typical D-type and TNO (dashed lines); b) Cometary nuclei which presented a faint coma during the observations.

concentrate closer in semimajor axis to Ferraz-Mello. In particular, the two asteroids that have the spectra most similar to FerrazMello are in this region.

\section{Comparative dynamics}

In this section we compare the dynamics of 5201(Ferraz-Mello) to the dynamics of a sample of asteroids and comets with orbits around the same position of (5201) in the $a-e$ plane (Fig. 4) and small inclinations. They have perihelions close to the orbit of Mars and aphelion near the orbit of Jupiter. The sample was selected from the lists of Jupiter family comets and of low-Tisserand asteroids posted in the web by Y. Fernandez (http://nexus.physics.ucf.edu/ yfernandez), with inclinations $i<10$ degrees. All objects in the interval $2.6<a<$ $3.8 \mathrm{AU}$ and $0.35<e<0.7$ with Tisserand parameter $<3$ are shown in Fig. 4. To have a minimum of dynamical consistency, only objects observed over more than 60 days were considered. These objects have quality codes 4 or smaller. The only exception is the asteroid 1999 SE10, with quality code 6 , which is the 


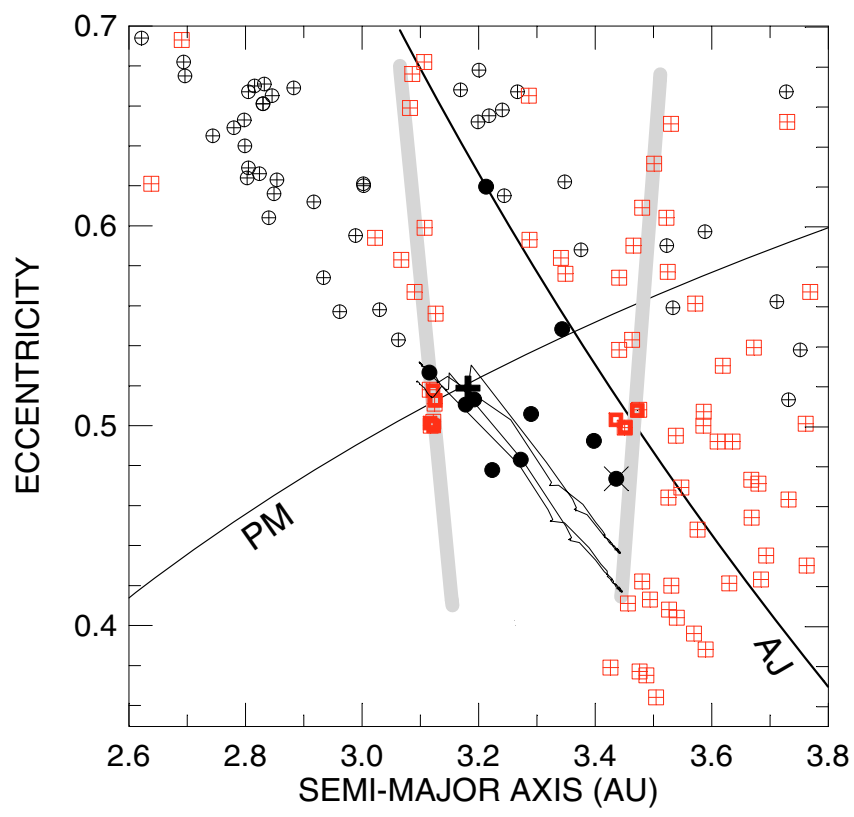

Fig. 4. Sample of asteroids and comets at the crossing of the lines PM (perihelion close to Mars) and AJ (aphelion close to Jupiter). The thick gray lines show the borders of the $2 / 1$ mean-motion resonance. Circles are asteroids and squares are comets. Bold symbols indicate the objects selected for study. The bold plus sign represents (5201) Ferraz-Mello. The evolution of this asteroid during the next 300 years is also shown.

closest object to the group with a known spectral class (class X of SMASS II) (Binzel et al. 2001). It is the only selected object with $e>0.6$.

The orbital evolution of the selected objects was simulated using a pure $N$-body code (RA 15) (Everhart 1985) including the object and all planets from Venus to Neptune. The initial heliocentric positions of the planets were taken from the Astronomical Almanac and those of the objects from the JPL Small-Body Database (http://ssd.jpl.nasa.gov/ sbdb.cgi). Since these data correspond to different epochs, in each case the positions of the 8 planets were propagated to the given epoch using the same code, with Mercury as the 8th body. The simulations were carried 4000 years forward and 4000 years backward. This time interval already exceeds the range in which the motion of chaotic objects such as (5201) can be predicted. The study of the divergence of neighboring orbits shows that the Lyapunov time of (5201) is not longer than 40 years. One consequence of this low Lyapunov time is that results from two simulations with slight different conditions may be completely different after a few centuries. This is the limit of predictability for (5201) and for several other objects in the studied sample. The figures show only the interval $0<t<4000$.

The results for the asteroids (3688) Navajo, 1992 RN1, 1999 SE10, 2000JV 60, 2002 NX46, 2002 XF46, 2003 XG, 2006 FH51, and 2006 FZ51 were very similar. All but one of them remain inside the $2 / 1$ resonance for the whole simulation and show characteristic patterns. They have frequent approximations to Mars, but not to Jupiter (they are protected by the resonance). The distance parameters $q, a, Q$ have only small fluctuations about constant values. The time of residence in the $2 / 1$ resonance of objects with dynamics similar to the ones that we have studied is known to be $10^{7}-10^{8}$ years (Roig et al. 2002) The Tisserand parameter presents a smooth variation. The behavior of the critical angle $\sigma=2 \lambda_{\text {Jup }}-\lambda-\varpi$ ( $\lambda$ is the mean longitude and $\varpi$ the longitude of the perihelion), the eccentricity, and the minimum

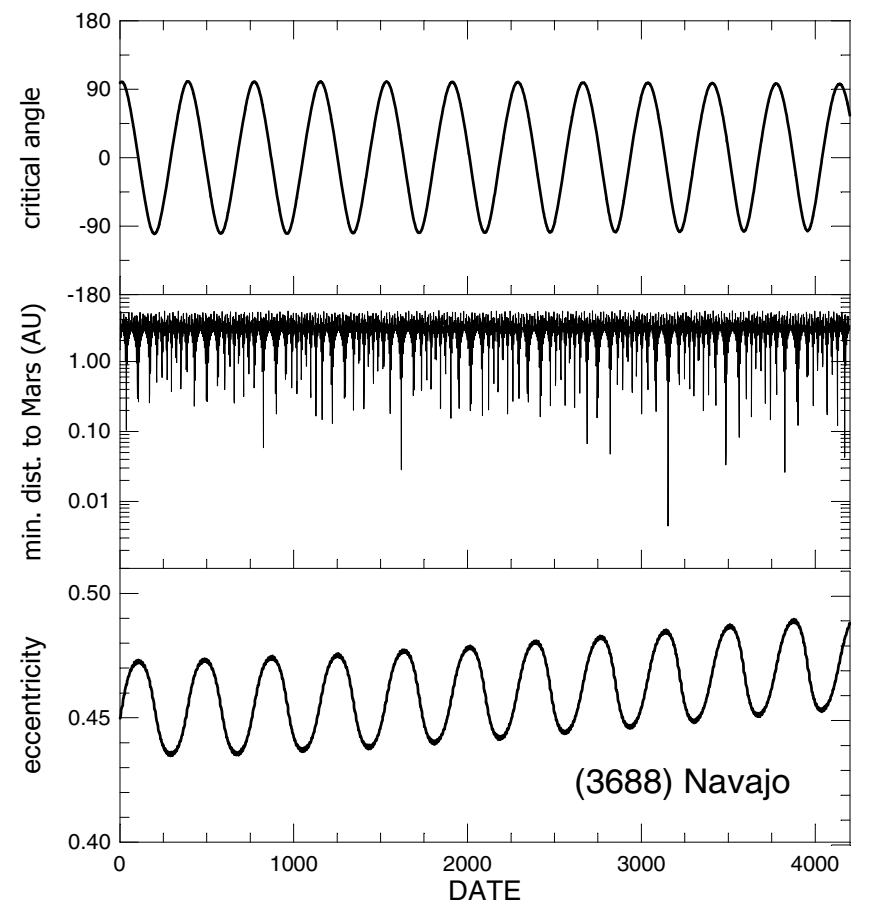

Fig. 5. Orbital evolution of (3688) Navajo. a) Libration around 0 of the critical angle $\left.\sigma=2 \lambda_{\text {Jup }}-\lambda-\varpi ; \mathbf{b}\right)$ minimum distancces of the asteroid to Mars; c) eccentricity.

distances to Mars are shown in Fig. 5. We selected the results for the asteroid (3688) Navajo as it shows the closest approximations to Mars $(0.004 \mathrm{AU}$ around $t=3100)$.

The behavior of the asteroid $2003 \mathrm{XG}$ is different. It is not in the resonance (The angle $\sigma$ circulates; It appears over the left resonance border in Fig. 4). It has several approaches to less than 1 UA to Jupiter, but not much closer, and it does not show any important variation on the time span of the simulations. One other asteroid has shown completely different behavior: 2005 YR50. It will be considered in the next section.

The results for the comets do not show the same uniformity as for the asteroids and have very different characteristics from those shown by the asteroids. They cluster in two groups, one near the left border of the resonance (9P/Tempel 1, 71P/Clark, $125 \mathrm{P} /$ Spacewatch, $\mathrm{P} / 2004 \mathrm{~K} 2 \mathrm{McNaught}$ ) and the other near the right border (57P/DuToit-Neujmin-Delporte, 57P/DuToitNeujmin-Delporte A and P/2004 T1 LINEAR-NEAT). They show the critical angle $\sigma$ alternating between libration and circulation and have frequent approaches to both Mars and Jupiter that result in strong variations in eccentricity and occasional jumps in the semi-major axis and in the Tisserand parameter. Both $125 \mathrm{P} /$ Spacewatch and $\mathrm{P} / 2004 \mathrm{~K} 2$ are the most regular in the sample, expending a large fraction of the simulated time span in the resonance or very close to it. The more irregular behaviors are shown by 57P/DuToit-Neujmin-Delporte and 57P/DuToitNeujmin-Delporte A.

The orbital evolution of 71P/Clark is shown in Fig. 6. One may distinguish the following points in the figure: (a) the comet is currently in the $2 / 1$ resonance, and has been there for many centuries (the first capture into the resonance occurs at $t \sim 0$, a time far beyond the predictability range of comet $71 \mathrm{P}$ even if only gravitational forces are considered); (b) the comet had in this period, many close approaches to Mars, reaching in one of them $0.055 \mathrm{AU}$ from the planet; (c) the comet has been protected from close approaches to Jupiter; but it is leaving the reso- 


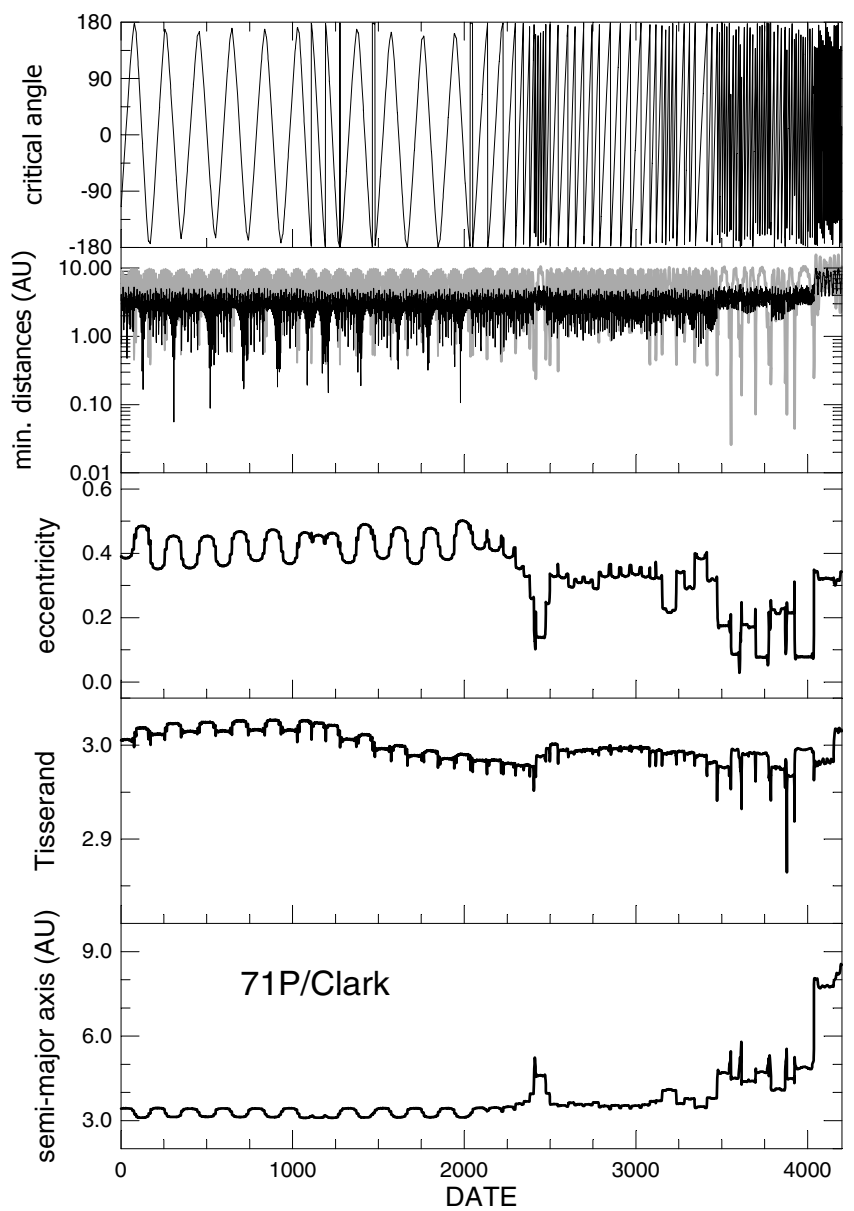

Fig. 6. Orbital evolution of $71 \mathrm{P} /$ Clark. a) Libration around 0 of the critical angle of the 2:1 resonance; $\mathbf{b}$ ) minimum distances of the body to Mars (black) and Jupiter (gray); c) eccentricity; d) Tisserand parameter.

nance, and the simulation shows approaches to Jupiter as close as $0.025 \mathrm{AU}$ in the next millennium; (d) the close approaches give rise to strong variations in $q, a, Q, e$, and in the Tisserand parameter; (e) after the close approaches to Jupiter, the solution corresponds to one comet moving in an orbit beyond Jupiter (seen at the bottom of figure).

The results of the simulations of the orbital evolution of 9P/Tempel 1 and P/2004 T1 LINEAR-NEAT have many localized similarities with the above one but their permanence in the resonance is much shorter in the first case, and longer in the other.

\section{The dynamics of (5201) Ferraz-Mello}

The results of the simulation of the orbital evolution of (5201) Ferraz-Mello are shown in Fig. 7. They are completely different from those found for the other asteroids in its neighborhood and present many similarities with the results of the simulations of 9P/Tempel 1 and 71P/Clark. (a) The asteroid is currently in the $2 / 1$ resonance, and is there since many centuries (the capture into the resonance is shown at $t \sim 1300$ ); b) the asteroid had in this period many close approaches to Mars (in some of them reached $0.01 \mathrm{AU}$ from the planet); c) the asteroid has been protected from close approaches to Jupiter, but it is leaving the resonance in $\sim 300$ years and the simulation shows approaches to Jupiter as close as $0.04 \mathrm{AU}$ in the next millennium; d) the close

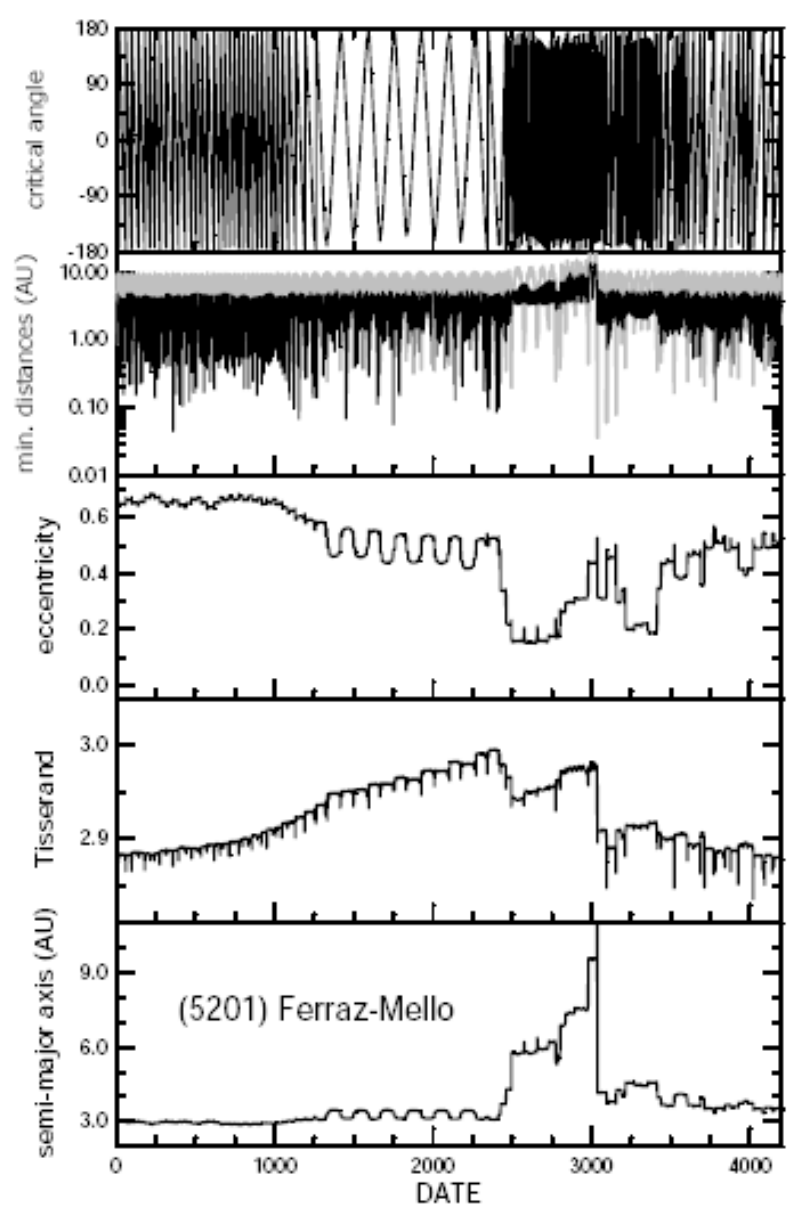

Fig. 7. Orbital evolution of (5201) 5201-dyn. Parameters as in Fig. 6.

approaches to Jupiter give rise to important variations in $q, a, Q$, $e$, and in the Tisserand parameter.

The dynamical similarity of (5201) Ferraz-Mello with 9P/Temple 1, 71P/Clark and P/2004 T1 LINEAR-NEAT (and the its dissimilarity with the other asteroids) is striking. This cannot be simply due to the proximity of them in the plane $a-e$. The initial conditions involve other elements not correlated with the position in the plane $a-e$ and they are responsible for the different behaviors observed. The evolutions of (5201) FerrazMello and the considered comets are marked by the fact that they lie inside the very stochastic layer enclosing the 2/1 resonance domain and this can be the ultimate determining factor of their dynamical similarity.

At last, we would like to mention asteroid 2005 YR50. It is close to the right border of the resonance and spikes were added to the symbol representing it in Fig. 4 to allow it to be identified. The plot of its present path in Fig. 4 (not shown) is similar to the path of (5201) Ferraz-Mello, except that it lies a little above in the graph but also extends from border to border, showing that this object is also inside the very stochastic layer of the resonance border. It is a dynamical twin of (5201) Ferraz-Mello. Its orbit is founded on a large arc (200 days) and has quality code 3 in the JPL Small-Body Database.

\section{Discussion}

We have obtained the first compositional characterization of the asteroid (5201) Ferraz-Mello, using broadband photometry in 
the SDSS filter system. The reflectance spectrum of this asteroid obtained this way is unusual, with a steep spectral gradient that is more like Centaurs and TNOs than to main belt D-type asteroids, but with a relative increase in the reflectance shortwards of $0.623 \mu \mathrm{m}$ that is not typically found in the former populations. A tentative explanation for this unusual spectrum is the presence of a faint dust coma, since cometary nuclei observed in the presence of a faint dust coma present similar spectral behavior. However, our images of (5201) Ferraz-Mello do not show any evidence of a coma, and an unusual composition cannot be ruled out as the culprit for the unusual spectrum of (5201) Ferraz-Mello.

We have also compared the dynamical evolution of (5201) Ferraz-Mello with a sample of asteroids and Jupiter family comets with orbits around the same position of (5201) in the $a-e$ plane. It is shown that the dynamics of the Jupiters family comets are significantly different from the asteroids in the region, even though the all the asteroid have $T_{\mathrm{j}}<3$, and the dynamical evolution of (5201) Ferraz-Mello is much more similar to the one of the comets.

Together, the unusual spectrum and the dynamical behavior of (5201) Ferraz-Mello strongly suggest that this object is in fact a dormant or extinct comet. This conclusion implies that some or all of the objects with spectra similar to (5201) Ferraz-Mello, which are all on typically asteroidal orbits, could also be extinct/dormant comets.

Acknowledgements. The authors acknowledge CNPq, FAPERJ, and FAPESP for diverse grants and fellowships.

\section{References}

Alvarez-Candal, A., \& Licandro, J. 2006, A\&A, 458, 1007

Babadzhanov, P. B., \& Kokhirova, G. I. 2007, Adv. Space Res., 39, 533

Benest, D., Bien, R., \& Rickman, H. 1985, in Astrophys. Space Sci. Library, 115, Dynamics of Comets: Their Origin and Evolution, ed. A. Carusi, \& G. B. Valsecchi, IAU Colloq., 83, 365
Binzel, R. P., Harris, A. W., Bus, S. J., \& Burbine, T. H. 2001, Icarus, 151, 139 Bowell, E., Mrkos, A., \& Everhart, E. 1984, IAU Circ., 3905, 2

Bus, S. J., \& Binzel, R. P. 2002, Icarus, 158, 146

Demeo, F., \& Binzel, R. P. 2008, Icarus, 194, 436

DeMeo, F. E., \& Binzel, R. P. 2006, in BAAS, 38, 581

Elst, E. W., Pizarro, O., Pollas, C., et al. 1996, IAU Circ., 6456, 1

Everhart, E. 1985, in Dynamics of Comets: Their Origin and Evolution, Proceedings of IAU Colloq., 83, held in Rome, Italy, June 11-15, 1984, ed. A. Carusi \& G. B. Valsecchi (Dordrecht: Reidel), Astrophys. Space Sci. Library, 115, ed. A. Carusi, \& G. B. Valsecchi, 185

Fernández, Y. R., Jewitt, D. C., \& Sheppard, S. S. 2005, AJ, 130, 308

Gunn, J. E. 1999, in BAAS, 31, 1418

Hahn, G., \& Rickman, H. 1985, Icarus, 61, 417

Howell, S. B. 1989, PASP, 101, 616

Hsieh, H. H., \& Jewitt, D. 2006, Science, 312, 561

Hsieh, H. H., Jewitt, D. C., \& Fernández, Y. R. 2004, AJ, 127, 2997

Ivezić, Ž., Tabachnik, S., Rafikov, R., et al. 2001, AJ, 122, 2749

Jenniskens, P. 2004, AJ, 127, 3018

Jewitt, D. C. 2002, AJ, 123, 1039

Jordi, K., Grebel, E. K., \& Ammon, K. 2006, A\&A, 460, 339

Juric, M., Ivezic, Z., Lupton, R., \& SDSS Collaboration. 2007, in American Astronomical Society Meeting Abstracts, 211, 56.05

Kresak, L. 1979, Dynamical interrelations among comets and asteroids, Asteroids, 289

Licandro, J., Alvarez-Candal, A., de León, J., et al. 2008, A\&A, 481, 861

Neese, C. 2006a, NASA Planetary Data System, EAR-A-5-DDR-TAXONOMYV5.0:TAXONOMY05_TAB, 48, 1

Neese, C. 2006b, NASA Planetary Data System, EAR-A-COMPIL-3-TNOCEN-COLOR-V3.0, 57

Rickman, H., Fernandez, J. A., \& Gustafson, B. A. S. 1990, A\&A, 237, 524

Roig, F., \& Gil-Hutton, R. 2006, Icarus, 183, 411

Roig, F., Nesvorný, D., \& Ferraz-Mello, S. 2002, MNRAS, 335, 417

Snodgrass, C., Lowry, S. C., \& Fitzsimmons, A. 2007, ArXiv e-prints, 712

Tholen, D. J., \& Barucci, M. A. 1989, in Asteroids II, ed. R. P. Binzel, T. Gehrels, \& M. S. Matthews, 298

Weissman, P. R., A'Hearn, M. F., Rickman, H., \& McFadden, L. A. 1989, in Asteroids II, ed. R. P. Binzel, T. Gehrels, \& M. S. Matthews, 880

Weissman, P. R., Bottke, Jr., W. F., \& Levison, H. F. 2002, Asteroids III, 669

Whipple, F. L. 1983, IAU Circ., 3881, 1

Williams, I. P., Ryabova, G. O., Baturin, A. P., \& Chernitsov, A. M. 2004, MNRAS, 355, 1171 